\title{
13. Sur les fonctions générales définies dans le domaine des nombres complexes.
}

\author{
Par Motokiti KoNDô. \\ Institut de Mathématiques, L'Université Impériale de Hokkaido, Sapporo. \\ (Comm. by M. Fujrwara, M.I.A., Feb. 12, 1936.)
}

Soient $D$ un domaine contenu dans le plan des nombres complexes et $\varphi(z)$ une fonction définie sur $D$ dont les valeurs appartiennent au domaine des nombres complexes. Il nous parait, alors, que $\varphi(z)$ ne soit qu'une combinaison linéaire de deux fonctions réelles $U(z)$ et $V(z)$, parties réelle et imaginaire de $\varphi(z)$, et que nous ne puissions établir les propriétés intéressantes sur $\varphi(z)$ sans admettre la régularité sur $\varphi(z)$. Cependant, d'après la caractéristique du domaine des nombres complexes, on peut établir des plusieurs propositions intéressantes sur $\varphi(z)$, pareilles aux théorèmes dans la théorie des fonctions analytiques d'une variable complexe." Dans cette Note et dans celles qui paraitront ulterieurement, nous allons montrer comment nous pouvons obtenir ces propositions sur $\varphi(z)$.

1. Le principe de M. Lindelöf. Nous allons donner tout d'abord le principe de M. Lindelöf et leurs applications sur les fonctions quelconques.

Théorème 1. Soient $D$ un domaine, $E$ un sous-ensemble non dense contenu dans $D$ qui jouit des propriétés suivantes: $1^{\circ} D-E$ est un domaine, $2^{\circ}$ Quels que soient le point frontière $z_{0}$ de $D$ et le nombre positif $\varepsilon$, il existe un voisinage $U\left(z_{0}\right)$ de $z_{0}$, tel que la frontière de $U\left(z_{0}\right)$ soit disjointe de $E$, et $\varphi(z)$ une fonction continue uniforme définie sur $D$, tel qu'on ait $3^{\circ}, \varphi(z)$ soit univalente au voisinage de chaque point de $D-E, 4^{\circ}$ On puisse définir un nombre positif $M$, comme il suit: quels que soient le point frontière $z_{0}$ de $D$ et le nombre positif $\varepsilon$, il existe un voisinage $U\left(z_{0}\right)$ de $z_{0}$, tel qu'on ait $|\varphi(z)| \leqq M+\varepsilon$ en tout point de $U\left(z_{0}\right) D$. Nous pouvons alors affirmer que l'inégalité $|\varphi(z)|<M$ est vérifiée en tout point de $D$.

Démonstration. Commençons par donner quelques terminologies geométriques. Nous dirons une courbe fermée généralisée une image localement topologique de l'ensemble de points $e^{i \theta}(0 \leqq \theta \leqq 2 \pi)$. Étant donnée une courbe fermée généralisée $C$ contenue dans le plan des nombres complexes, nous appellerons la partie intérieure de $C$ l'ensemble de tous les points $z$ du plan tels qu'on ne puisse joindre $z$ et $\infty$ par une courbe disjoint de $C$ et que $z$ n'appartienne pas à $C$. On peut alors énoncer le lemme suivant sur les composantes $E^{*}$ de $E$.

1) Les fonctions non régulières ont été étudiées par MM. S. Shimizu, K. Yoshida,

S. Kakutani, H. Grötsch, S. Stöilow, et M. Lavrentieff, voir p. ex.,

S. Shimizu：輓近函數論 (岩波㳟座).

K. Yoshida et S. Kakutani：全國林上數學談話會 40,41, 49, 52 號.

H. Grötsch: Leipziger Berichte, 80 (1928).

S. Stöilow : Annales Sc. d. l'Ec. Norm. Sup. (3) 45 (1928).

M. Lavrentieff : Comptes Rendus, 200 (1935). 
Lemme. Étant donnée une composante $E^{*}$ de $E$, on peut définir un polygone $\pi$ dans le domaine $D-E$, comme il suit : $1^{\circ} E^{*}$ est contenue dans la partie intérieure de $\pi .2^{\circ} \varphi\left(E^{*}\right)$ est contenue dans la partie intérieure de la courbe fermée généralisée $\varphi(\pi)$.

Maintenant nous allons démontrer d'abord que $|\varphi(z)|$ n'atteint le maximum en aucun point de $D$. Pour cela, nous supposerons que $|\varphi(z)|$ atteigne le maximum en un point $z_{0}$ de $D$. Lorsque $z_{0}$ appartient à $D-E \varphi(z)$ est univalente au voisinage de $z_{0}$, ce qui contredit l'hypothèse de $z_{0}$. Ensuite, lorsque $z_{0}$ appartiennent à $E$, il existe une composante $E^{*}$ de $E$ qui contient le point $z_{0}$, ce qui est contradictoire avec le lemme. Donc, $|\varphi(z)|$ n'atteint pas le maximum dans $D$, ce qui donne le théorème 1 .

C. Q. F. D.

Théorème 2. Soient $D$ un domaine, $E$ un sous-ensemble de $D$ qui jouit des propriétés $1^{\circ}$ et $2^{\circ}$ du théorème $1, F$ un sous-ensemble fermée de la frontière de $D, \varphi(z)$ une fonction continue uniforme définie sur $D$, qui jouit des propriétés suivantes: $1^{\circ} \mathrm{Au}$ voisinage de tous les points de la frontière de $D$ qui n'appartiennent pas à $F, \varphi(z)$ est inférieure en module à tout nombre supérieur à un certain nombre positif $\boldsymbol{M}$, $2^{\circ}$ Il existe une fonction continue uniforme $\omega(z)$ définie en tout point de $D$, ayant les propriétés suivantes : $\omega(z)$ ne s'annule pas dans $D$; son module y est au plus égal à 1; quel que soit le nombre positif $\lambda$, $\varphi(z)\{\omega(z)\}^{\lambda}$ est univalente au voisinage de chaque point de $D-E$ et inférieure à tout nombre supérieure à $M$ au voisinage de tout point de $F$. Nous pouvons alors affirmer que l'inégalité $|\varphi(z)| \leqq M$ est verifiée en tout point de $D$.

2. Le principe du maximum pour les fonctions harmoniques. D'après les théorèmes 1 et 2 , on peut donner une extension du principe du maximum pour les fonctions harmoniques comme il suit. Pour cela, donnerons d'abord la définition. Nous appellerons une fonction harmonique au sens large définie dans $D$ toute fonction qui peut être défini comme la partie réelle d'une fonction continue uniforme définie dans $D$ et satisfaisant aux conditions $1^{\circ}, 2^{\circ}$, et $3^{\circ}$ du théorème 1 . Nous avons alors

Théorème 3. Soient $D$ un domaine et $G(z)$ une fonction continue uniforme définie dans $D$ et satisfaisant aux conditions suivantes: $1^{\circ}$ $G(z)$ est harmonique au sens large dans $D, 2^{\circ}$ On peut définir un nombre positif $M$ comme il suit: quels que soient le point frontière $z_{0}$ de $D$ et le nombre positif $\varepsilon$, il existe un voisinage $U\left(z_{0}\right)$ de $z_{0}$, tel qu'on ait $G(z) \leqq M+\varepsilon$ en tout point de $U\left(z_{0}\right) D$. Nous pouvons alors affirmer que l'inégalité $G(z) \leqq M$ est verifiée en tout point de $D$.

3. Le principe de la majorante harmonique. Comme nous avons le principe du maximum pour les fonctions harmoniques, on peut établir une extension du principe de la majorante harmonique, comme il suit.

Théorème 4. Soient $D$ un domaine, $\varphi(z)$ une fonction continue uniforme définie dans $D, \chi(z)$ une fonction continue uniforme définie dans $D$ et satisfaisant aux conditions suivantes: $1^{\circ}$ Quel que soit le polynome $P(t)$, on peut définier un sous-ensemble $E=E(P(t))$ de $D$ tel que $E$ satisfait aux conditions $1^{\circ}$ et $2^{\circ}$ du théorème 1 et que $P(\chi(z)) \varphi(z)$ 
est univalente au voisinage de chaque point de $D-E, 2^{\circ} \log |\varphi(z)|-G(z)$ est inférieure à tout nombre positif au voisinage de tout point de la frontière de $D$, où $G(z)$ est la partie réelle de $\chi(z)$. Dans ces conditions, l'inégalité $\log |\varphi(z)| \leqq G(z)$ est vérifiée en tout point de $D$.

4. Les théorèmes de Liouville et de Picard. Enfin, nous allons donner une extension des théorèmes de Liouville et de Picard comme application du théorème 1 et 2.

Théorème 5. Soient $D$ un domaine $|z|<\infty, \varphi(z)$ une fonction continue uniforme définie dans $D$ et satisfaisant à la condition : quels que soient les polynomes $P(t)$ et $Q(t)$, la fonction $P(z) Q(\varphi(z))$ est univalente au voisinage de chaque point de $D$ sauf les points formant un ensemble $E(P, Q)$ qui satisfait aux conditions $1^{\circ}$ et $2^{\circ}$ du théorème 1 . Dans ces conditions, $\varphi(z)$ est non borné dans $D$.

Théorème 6. Soit $\varphi(z)$ une fonction continue uniforme définie en tout nombre complexe fini: $|z|<\infty$ et satisfaisant aux conditions du théorème 5. Alors, $\varphi(z)$ prend tous les nombres complexes finis sauf peut-être un.

Remarque. Disons qu'une fonction jouit de la propriété de Lindelöf, lorsqu'elle appartient à la famille de fonctions pour lesquelles le principe de M. Lindelöf est applicable. Alors, comme la condition de l'univalence dans les théorèmes 2-6 est introduite pour faire directement l'application du théorème 1 , on peut remplacer cette condition par celle de la jouissance de la propriété de Lindelöf.

Le 10 Janvier 1936. 